\title{
A VLBI study of the wind-wind collision region in the massive multiple HD 167971
}

\author{
J. Sanchez-Bermudez ${ }^{1,2,3}$, A. Alberdi ${ }^{4}$, R. Schödel ${ }^{4}$, W. Brandner ${ }^{2}$, R. Galván-Madrid ${ }^{5}$, J. C. Guirado ${ }^{6,7}$, \\ R. Herrero-Illana ${ }^{1}$, C. A. Hummel ${ }^{8}$, J. M. Marcaide ${ }^{6}$, and M. A. Pérez-Torres ${ }^{4, \star}$ \\ ${ }^{1}$ European Southern Observatory, Alonso de Córdova, 3107 Vitacura, Santiago de Chile, Chile \\ 2 Max-Planck-Institut für Astronomie, Königstuhl 17, 69117 Heidelberg, Germany \\ 3 Instituto de Astronomía, Universidad Nacional Autónoma de México, Apdo. Postal 70264, Ciudad de México 04510, Mexico \\ e-mail: joelsb@astro.unam.mx \\ ${ }^{4}$ Instituto de Astrofísica de Andalucía (IAA-CSIC), Glorieta de la Astronomía S/N, 18008 Granada, Spain \\ 5 Instituto de Radioastronomía y Astrofísica (IRyA), UNAM, Apdo. Postal 72-3 (Xangari), Morelia, Michoacán 58089, Mexico \\ 6 Departament d'Astronomia i Astrofísica, Universitat de Valencia, C. Dr. Moliner 50, 46100 Burjassot, Valencia, Spain \\ 7 Observatori Astronòmic, Universitat de València, Parc Científic, C Catedrático José Beltrán 2, 46980 Paterna, València, Spain \\ 8 European Southern Observatory, Karl-Schwarzschild-Straße 2, 85748 Garching, Germany
}

Received 15 November 2018 / Accepted 29 January 2019

\begin{abstract}
Context. Colliding winds in massive binaries are able to accelerate particles up to relativistic speeds as the result of the interaction between the winds of the different stellar components. HD 167971 exhibits this phenomenon which makes it a strong radio source. Aims. We aim at characterizing the morphology of the radio emission and its dependence on the orbital motion, traced independently by near-infrared (NIR) interferometry of both the spectroscopic binary and the tertiary component comprising HD 167971.

Methods. We analyze 2006 and 2016 very long baseline interferometric data at $C$ and $X$ bands. We complement our analysis with a geometrical model of the wind-wind collision region and an astrometric description of the system.

Results. We confirm that the detected nonthermal radio emission is associated with the wind-wind collision region of the spectroscopic binary and the tertiary component in HD 167971. The wind-wind collision region changes orientation in agreement with the orbital motion of the tertiary around the spectroscopic binary. The total intensity also changes between the two observing epochs in a way that is inversely proportional to the separation between the two components, with a negative-steep spectral index typical of an optically thin synchrotron emission possibly steepened by an inverse Compton cooling effect. The wind-wind collision bow-shock shape and its position with respect to the stars indicates that the wind momentum from the spectroscopic binary is stronger than that of the tertiary. Finally, the astrometric solution derived for the stellar system and the wind-wind collision region is consistent with independent Gaia data.
\end{abstract}

Key words. radiation mechanisms: non-thermal - techniques: high angular resolution - techniques: interferometric binaries: general - stars: massive - stars: mass-loss

\section{Introduction}

Massive binaries (and higher-degree multiple systems) made of O-type and/or Wolf-Rayet (WR) stars are known to produce stellar wind collisions and have shown their capability to accelerate particles up to relativistic speeds. The individual stellar winds collide and form shocks that define the windwind collision region (WWCR). Within this region, electrons are accelerated to relativistic velocities, emitting synchrotron radiation which is detected as nonthermal radio emission. This emission is characterized by a negative spectral index ${ }^{1}$ and a high brightness temperature (see e.g., Eichler \& Usov 1993; Williams et al. 1990; White \& Becker 1995; Dougherty et al. 1996, 2003; Dougherty \& Williams 2000; Pittard \& Dougherty

\footnotetext{
* Visiting Scientist: Departamento de Física Teórica, Facultad de Ciencias, Universidad de Zaragoza, Spain.

$1 S_{v} \propto v^{\alpha} ;$ it is assumed that the flux density, $S_{v}$, is proportional to the frequency of observation, $v$, scaling with a power-law defined by the spectral index $\alpha$.
}

2006; Pittard et al. 2006; Blomme et al. 2007; Montes et al. 2009, 2015.) Most of the stellar nonthermal radio emitters are multiple systems with at least one WR star. There are only a few known nonthermal systems composed exclusively by $\mathrm{O}$ stars (see e.g., Rauw 2004; Benaglia \& Koribalski 2007). The study of these systems is necessary to understand the properties of their wind-wind interactions. In the last decade several individual studies have been carried out on stellar radio sources like $9 \mathrm{Sgr}$ (Blomme \& Volpi 2014), HD 168112 (De Becker et al. 2004; Blomme et al. 2005), or Cyg OB2 No. 9 (van Loo et al. 2008) among others, characterizing the radio emission in terms of the stellar parameters of the sources, and of the wind-wind collision region.

HD 167971, at a distance of $1.8 \mathrm{kpc}$ (De Becker et al. 2012), is one of the brightest synchrotron stellar radio emitters (Bieging et al. 1989). It is a hierarchical triple system (Leitherer et al. 1987), which consists of a spectroscopic binary (SB), with a period of $\sim 3.3$ days, and a tertiary (T) component moving on a wider orbit, with a period of $\sim 21.4$ years. The spectral types of the stars are O7.5III (Aa), O9.5III (Ab), and 
O8I (B) ${ }^{2}$. Near-infrared (NIR) interferometric observations obtained with AMBER, PIONIER and GRAVITY at the Very Large Telescope Interferometer (VLTI) and reported by De Becker et al. (2012) and Le Bouquin et al. (2017) demonstrated that the SB and T are gravitationally bound, with a projected separation that varies from 8 to 15 milliarcsec (mas). The angular separation between the components of the SB is of the order of 0.1 mas, and cannot be spatially resolved with the VLTI nor with radio very long baseline interferometry (VLBI). Therefore, HD 167971 appears as a binary system (SB-T) in the nearinfrared interferometric data.

Figure 1 presents the best-fit orbit of $\mathrm{T}$ around the SB reported by Le Bouquin et al. (2017). This solution is in agreement with previous estimates reported by the light-curve analyses of Blomme et al. (2007) and Ibanoglu et al. (2013). The VLA and ATCA radio light curves studied by Blomme et al. (2007) presented a periodicity consistent with the reported orbital period of the tertiary, and the emission showed a negative spectral index, thereby confirming its nonthermal nature. These findings supported the hyphothesis that the nonthermal radio emission is produced in the wide orbit, probably in the adiabatic wind colliding region between the SB and T. The difference of the light-curve profiles between $6 \mathrm{~cm}(5 \mathrm{GHz})$ and $20 \mathrm{~cm}(1.5 \mathrm{GHz})$ suggests that the emitting region is relatively extended.

In addition to the observed radio emission, De Becker et al. (2005) reported X-Ray (thermal) emission at energies of $2-4 \mathrm{keV}$ that correspond to a high-temperature plasma $\left(2.3-4.6 \times 10^{7} \mathrm{~K}\right)$, which is typical of pre-shock winds near their terminal velocity. This fact is in agreement with a wind-wind interaction scenario with strong adiabatic shocks, and a phase variability scaling with the inverse of the distance between the SB and the T. These characteristics make HD 167971 a unique target to test the physics of wind-wind collisions in $\mathrm{O}$ stars.

Here, we report new HD $167971 X-(3.5 \mathrm{~cm} ; 8.4 \mathrm{GHz})$ and $C$-band $(6.0 \mathrm{~cm} ; 5 \mathrm{GHz})$ observations with the Very Long Baseline Array (VLBA) obtained in 2016, complemented with archival VLBA data at the same frequencies from 2006. Section 2 presents our observations and data reduction. In Sect. 3 the analysis of the radio emission and our results are presented. Subsequently, in Sect. 4 we present the conclusions of our work.

\section{Observations and data reduction}

We performed VLBA observations of HD 167971 on August 13, 2016, at $C$-band and on August 14, 2016 at $X$-band. We used a sustained data-recording rate of $2 \mathrm{Gbit} \mathrm{s}^{-1}$ in two-bit sampling. Each frequency band was split into eight intermediate frequencies (IFs) of $32 \mathrm{MHz}$ bandwidth each, for a total synthesized bandwidth of $256 \mathrm{MHz}$. Each IF was in turn split into 32 channels of $1.0 \mathrm{MHz}$ bandwidth. The data were corrected for Earthorientation and ionospheric effects. The source J1751+0939 was used as bandpass calibrator and, the observations were phasereferenced to the source J1818-1108. Since this phase calibrator is not compact, all the fringe-fitting solutions were corrected from its structure.

The data were correlated at the NRAO data processor using an averaging time of $2 \mathrm{~s}$. We performed standard a-priori gain calibration using the measured gains and system temperatures of each antenna. This calibration, as well as the data inspection and

\footnotetext{
2 The labels $\mathrm{Aa}, \mathrm{Ab}$ and $\mathrm{B}$ follows the IAU component designation. However, in this work we would refer to the system $\mathrm{Aa}-\mathrm{Ab}$ as the spectroscopic binary, $\mathrm{SB}$, and the component $\mathrm{B}$ as the tertiary, $\mathrm{T}$.
}

editing, were done within the NRAO Astronomical Image Processing System (AIPS). No self-calibration was performed on the data since the peaks of emission were too faint. We also used AIPS to produce the images of both the reference source and target. Additionally, we analyzed archival VLBA observations performed on August 4, 2006, half the orbital period of the system earlier. A total synthesized bandwidth of $32 \mathrm{MHz}$ was used, hence rendering the observations of lower sensitivity than those of 2016. The same calibrator source was used, and the fringefitting solutions were also corrected for the calibrator structure. Figure 2 shows the images of both epochs that were recovered by applying natural weighting to the data. Table 1 displays the main characteristics of the reconstructed maps.

\section{Analysis and results}

\subsection{Radio map analysis}

The reported VLBA observations image the nonthermal radio emission of the WWCR. Figure 2 displays the radio maps, which show that the emission is resolved and consists of an elongated structure that changes depending on the observing epoch. The observing epochs correspond to half an orbital period, and show radio structures that have rotated by almost $90^{\circ}$. Considering the orbital solution from Le Bouquin et al. (2017), and the radio images, we confirm that the long-axis of the radio emission for every frequency and epoch - is perpendicular to the line that connects the SB with the T. Our observations therefore support the hypothesis that the observed radio emission corresponds to the wind-wind collision region between the SB and the T. The following structural properties are highlighted.

- The interacting point of the two winds could be characterized assuming that the WWCR is driven by the wind momenta ratio of the interacting stars $\left(\beta=\dot{M}_{\mathrm{T}} \nu_{\infty, \mathrm{T}} / \dot{M}_{\mathrm{SB}} \nu_{\infty, \mathrm{SB}}\right)$. In this case, a bowshock-like structure is formed at the shock front between the two winds. This bowshock has its tails pointing towards the star with the smaller wind momentum. In the 2006 epoch, a similar bow-shock (arc-like) feature is observed with small tails pointing towards the $\mathrm{T}$, implying that the wind momentum of the SB is larger.

- The fact that the bowshock is better defined in the 2006 map is consistent with the projected orbital position of the $\mathrm{T}$ at the predicted orbital phase, $\phi$. In the 2006 data $^{3}\left(\phi_{2006}=-0.1\right)$, we are observing the parabolic shock profile with a small inclination angle ${ }^{4}\left(i=14.5^{\circ}\right)$ from the plane of the sky, while in the 2016 epoch $\left(\phi_{2016}=0.37\right.$; where the $\mathrm{T}$ is behind the SB in our line-of-sight) we are observing the projected apex of the parabolic bowshock pointing towards us $\left(i=-152.8^{\circ}\right)$ and thus its morphology resembles an elongated Gaussian instead of a parabolic arc (see Fig. 1 for a visual identification of the position of the $\mathrm{T}$ at the two analyzed epochs).

- In the $X$-band maps, we identified an additional compact structure to the bowshock. In the 2006 epoch it can be observed as a small, elongated appendix to the northwest of the central emission peak. In the 2016 map, a similar appendix is also observed to the northeast of the central peak, and the peak itself appears to be shifted from the center of the emission. Possible explanations for this include (i) the interaction of

\footnotetext{
3 For the current work, we assume epoch $T_{0}=2454736.5 \mathrm{JD}$ as reference for the orbital phases.

4 Inclination angle, $i$, is measured between the bowshock (rotational) symmetry axis to the plane of the sky. This angle increases clockwise in a plane orthogonal to the plane of the sky in direction of the observer's line-of-sight.
} 

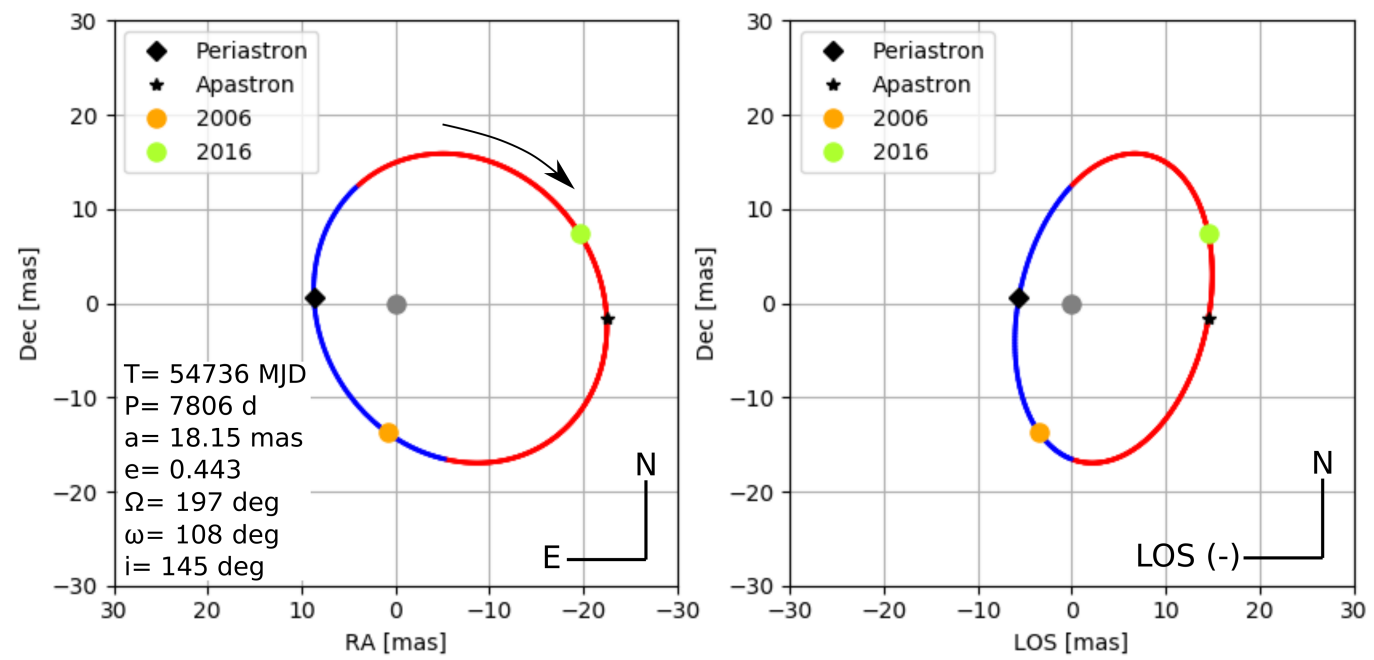

Fig. 1. Left panel: orbit of the SB-T system projected in the plane of the sky. Right panel: orbit of the SB-T system in a plane orthogonal to the plane of the sky, formed by the observer's line-of-sight and north. The orbital solution was obtained from Le Bouquin et al. (2017) and the mean orbital parameters are labeled on the image. The phases of the $\mathrm{T}$ at the two VLBI epochs reported in this work are shown with orange and green dots in the orbital solution. The black arrow shows the projected clock-wise motion of the T. The periastron and apastron are also displayed in the panels. The portion of the orbit in which the $\mathrm{T}$ is in front of the SB, in our line-of-sight, is displayed in blue, while the portion of the orbit in which the $\mathrm{T}$ is behind the $\mathrm{SB}$ is labeled in red.

Table 1. VLBA observations.

\begin{tabular}{lcccc}
\hline \hline & \multicolumn{2}{c}{ 2006 epoch } & \multicolumn{2}{c}{2016 epoch } \\
\cline { 2 - 5 } Parameter & $X$-band & $C$-band & $X$-band & $C$-band \\
\hline Beam $F W H M(\mathrm{mas})$ & $4.6 \times 2.4$ & $10.8 \times 6.9$ & $4.38 \times 2.11$ & $7.36 \times 3.54$ \\
Beam PA $(\mathrm{deg})$ & 4.0 & 42.0 & -5.0 & 7.0 \\
rms-noise $\left(\mu \mathrm{Jy}\right.$ beam $\left.^{-1}\right)$ & 134.0 & 196.0 & 40.0 & 45.0 \\
Total $S_{v}(\mathrm{mJy})$ & 5.324 & 12.314 & 2.817 & 7.316 \\
Peak's $S_{v}\left(\mathrm{mJy} \mathrm{beam}^{-1}\right)$ & 1.507 & 5.604 & 0.423 & 0.169 \\
\hline
\end{tabular}

nonspherical/clumpy winds or (ii) the interaction of windmomenta of similar magnitude that could disturb the profile from the parabolic bow-shock solution (see Fig. 2 in Canto et al. 1996).

- We obtained spectral index maps of HD 167971 between 8.4 and $5 \mathrm{GHz}$ (see Appendix A). The spectral index corresponds to nonthermal emission, with a value of $\alpha \sim-1.1$ quite constant along the whole shocked region, suggesting that the Fermi acceleration mechanism is efficient. Dougherty et al. (2003) show that a synchrotron spectrum without losses goes as $\propto v^{-0.5}$. However, HD 167971 has a steeper spectral index, which suggests a possible attenuation of the synchrotron emission due to several physical mechanisms (see below).

Based on typical stellar parameters for $O$ stars, Blomme et al. (2007) determined that the radio photosphere of optical depth unity at $5 \mathrm{GHz}$ is $\leq 2000 R_{\odot}$ (at $8.4 \mathrm{GHz}$, it is smaller), shorter than the minimum linear separation between the eclipsing binary and the tertiary component of the system $\left(\sim 4000 R_{\odot}\right)$. Therefore, the observed WWCR should be outside the radio photosphere, with a negligible free-free absorption, showing the intrinsic optically thin synchrotron emission at all orbital phases. It is predicted that at frequencies lower than $5 \mathrm{GHz}$ (e.g., at $1.4 \mathrm{GHz}$ ), the radio photosphere is much more extended. Therefore, the radio emission of the WWCR is embedded in the radio photosphere for a large fraction of the orbit, resulting in spectral index and turnover frequency changes of the radio emission along the orbit, which are not detectable at 5 or $8.4 \mathrm{GHz}$. Under these assumptions, attenuation of the synchrotron emission at the observed frequencies due to the Razin effect (Dougherty et al. 2003) is also negligible.

We suspect that the steepness of $\alpha$ is caused by inverse Compton cooling, which is more efficient for electrons with higher energies. According to the model presented by Pittard et al. (2006), inverse Compton rapidly decreases the energy of electrons close to the central volume of the WWCR, confining only a portion of relatively energetic electrons in layers near the shocks, which in turn results in a steeper spectrum at high frequencies.

\subsection{Bow-shock model}

Since the projected bow-shock profile in the 2006 epoch does not exhibit long tails, despite the small inclination angle from the plane of the sky, we suspect that the wind-momenta ratio of the SB and T are quite similar. To estimate the separation of the WWCR and $\beta$, we used the algebraic solution of Canto et al. (1996) to define the shock profile of two interacting winds:

$R=D \sin \left(\theta_{\mathrm{SB}}\right) \csc \left(\theta_{\mathrm{SB}}+\theta_{\mathrm{T}}\right)$,

where in our system $R$ corresponds to the bow-shock distance from the T, and $D$ is the separation between the SB and the T. The distance from the $\mathrm{T}$ to the apex of the bowshock is known as the stand-off distance $R_{0}$. The angles $\theta_{\mathrm{T}}$ and $\theta_{\mathrm{SB}}$ are the different 

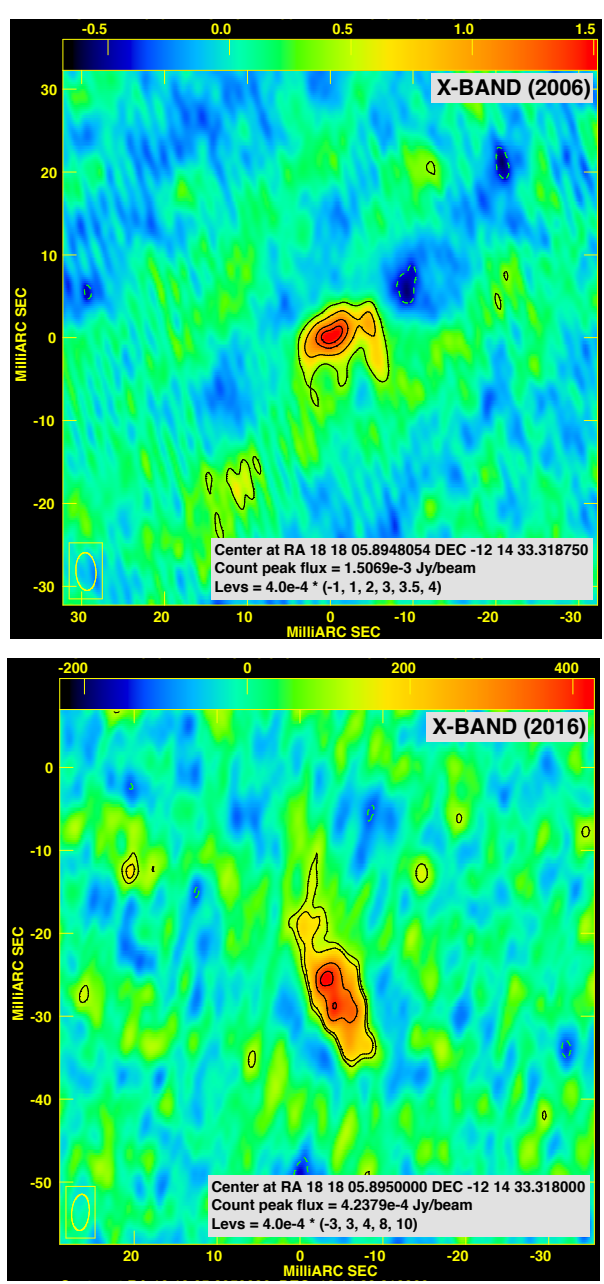
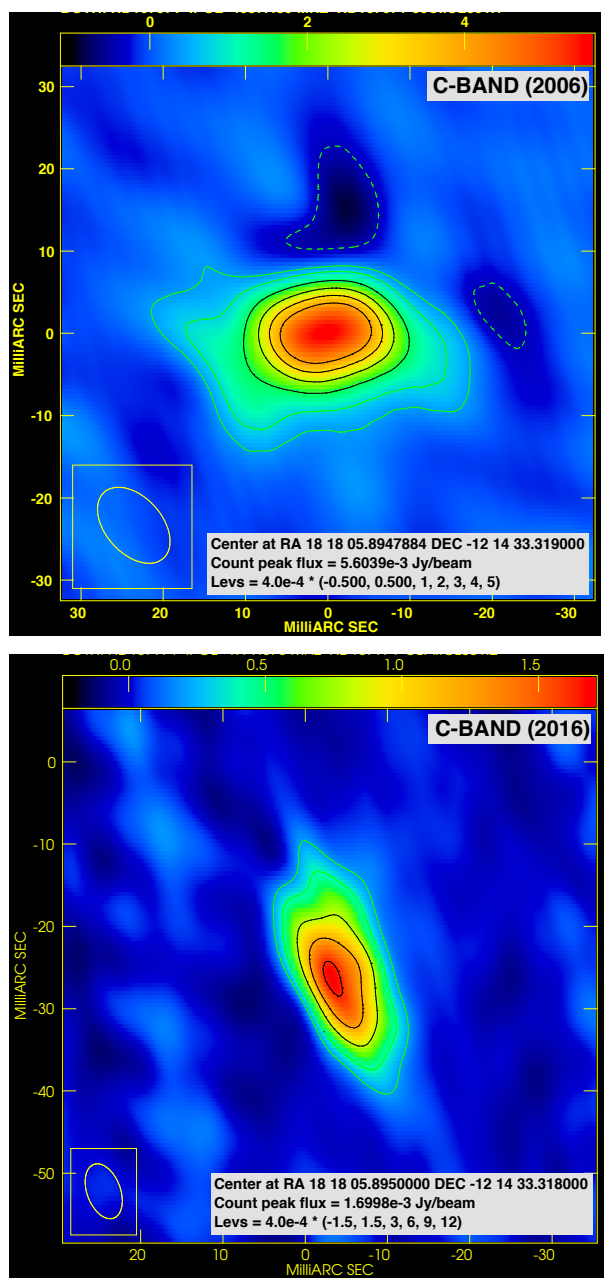

Fig. 2. Maps of the nonthermal radio emission observed in HD 167971. The radio bands and epochs are shown in each of the image panels. The synthesized beam, coordinates of the field center, peak-flux, and contour levels are also displayed in each of the panels. half-opening angles between the line that connects the SB and the $\mathrm{T}$ with the profile of the wind-wind shock front. The halfopening angles depend on $\beta$, as follows:

$\theta_{\mathrm{SB}} \cot \left(\theta_{\mathrm{SB}}\right)=1+\beta\left(\theta_{\mathrm{T}} \cot \left(\theta_{\mathrm{T}}\right)-1\right)$.

Since the wind-wind shock profile is more homogeneous in the $C$-band, we used the 2006 map to model the bow-shock profile with the previous geometrical solution through the following method.

- We mapped the position of the maximum of the brightness distribution of the source for each column of pixels perpendicular to the bow-shock structure in the $2006 C$-band image. A prior rotation according to the orbital position, and image centering in a common reference frame were applied to the original map. We restricted our analysis to the structure with a brightness larger than $20 \%$ of the flux peak. To map the bow-shock profile, a Gaussian was fit to each nonzero element per column of pixels in the image. To estimate the errors in the position of the bow-shock profile, the standard-deviation of the Gaussian fit was used with a weight proportional to the number of pixel elements fitted.

- Once the profile was estimated, it was compared with the projected canonical solution of the wind-wind bowshock obtained from a Monte-Carlo Markov-chain (MCMC) model using emcee (Foreman-Mackey et al. 2013). Our MCMC model evaluates values of $\beta$ between 0.3 and 0.9 , smaller $\beta$ values would produce a very close bowshock with long tails, while higher ones would produce a completely open bowshock. The model was run with ten independent chains of 1000 steps each. For every iteration, the distance between the $\mathrm{T}$ and the shock front was adjusted for both the model and data, according to the following expression.

$R_{0}=\frac{\beta^{1 / 2} D}{1+\beta^{1 / 2}}$.

Although the stand-off distance is variable in this procedure since the half-opening angle of the bowshock depends on the wind momenta ratio, we found the most probable model at a $\beta$ and a stand-off distance that match the half-opening angle of the extracted profile better. The probability density function of the MCMC exhibits a clear peak at $\beta=0.48 \pm 0.07$ (see Fig. 3), which locates the shock front at a projected $R_{0}=0.41 D \pm 0.02 D$ from the T, with $D$ being the separation between the SB and the $\mathrm{T}$ across the orbital path. The $\beta$ value therefore corresponds to a half-opening angle $\theta_{\mathrm{T}}=103^{\circ} \pm 3^{\circ}$ of the bow-shock profile.

The used model is a solution for a radiative wind-wind collision shock. However, it is quite probable for the shock in HD 167971 to be adiabatic. Therefore, we compared the model solutions for adiabatic shocks described in Gayley (2009) and Pittard \& Dawson (2018). The model for an adiabatic shock without mixing requires a $\beta$ of $\sim 0.39$ (notice that this value is within $2 \sigma$ of the reported $\beta$ value for the radiative shock) to generate a half-opening angle of $\theta_{\mathrm{T}} \sim 103^{\circ}$. However, the situation changes when there is an adiabatic shock with mixing. In this case, the value of $\beta$ depends on the ratio of the terminal velocity of the winds, $u=v_{T} / v_{S B}$. The bigger the value of $u$, the smaller 


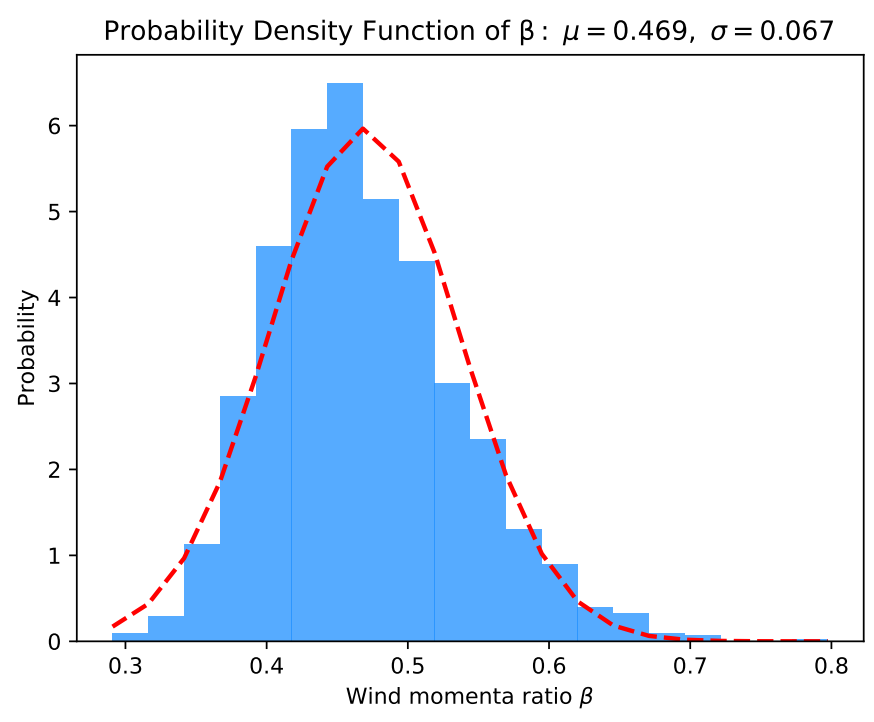

Fig. 3. Probability density distribution of $\beta$. The values were obtained from the MCMC minimization of the geometrical modeling applied to the wind-wind bow-shock profile of HD 167971.

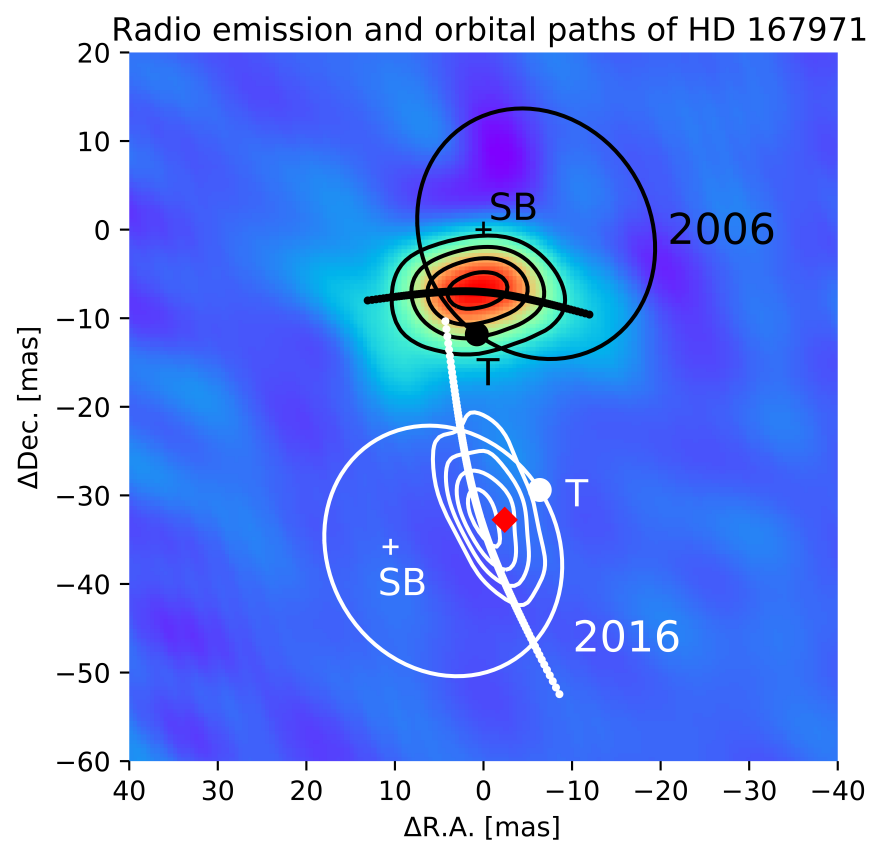

Fig. 4. Astrometric position of the SB-T system and the $C$-band radio emission of the 2006 and 2016 epochs. The image is centered at $\mathrm{RA}=274.5245616$, Dec $=-12.2425868$. The different stellar components and the radio emission are represented in black for the 2006 epoch, and in white for the 2016 one. The SB is represented by a cross, the $\mathrm{T}$ with a dot. The projected orbital path is shown with a solid line. The $2006 C$-band radio-emission is in colour scale contoured in black (representing 30, 50, 70, and 90\% of the peak), while the 2016 one is only displayed in white contours. The thick-solid lines represent the 2D bow-shock profile from our geometrical model. The red diamond shows the Gaia astrometric measurement for the system in the 2016 epoch.

the values of $\beta$ required to keep the observed half-opening angle (see Fig. B.1). In turn, the position of the shock relative to the two stellar components would be modified considerably. It is beyond the scope of this work to explore these models in detail. However, they should be taken into account for future studies.
Table 2. HD 167971 astrometry.

\begin{tabular}{|c|c|c|c|c|}
\hline \multicolumn{5}{|c|}{ VLBI coordinates of the emission's peak } \\
\hline Epoch & RA & $\sigma_{\mathrm{RA}}$ & Dec & $\sigma_{\text {Dec }}$ \\
\hline 2006.59 & $274.5245618^{\circ}$ & 0.4 mas & $-12.2425887^{\circ}$ & $0.2 \mathrm{mas}$ \\
\hline 2016.61 & $274.5245618^{\circ}$ & 1.9 mas & $-12.2425957^{\circ}$ & 0.9 mas \\
\hline \multicolumn{5}{|c|}{ SB relative position from the VLBI coordinates ${ }^{(a)}$} \\
\hline Epoch & $\Delta \mathrm{RA}$ & $\sigma_{\Delta \mathrm{RA}}$ & $\Delta \mathrm{Dec}$ & $\sigma_{\Delta \mathrm{Dec}}$ \\
\hline 2006.59 & -0.4 mas & 0.4 mas & 7.0 mas & $0.3 \mathrm{mas}$ \\
\hline 2016.61 & -10.0 mas & $2.0 \mathrm{mas}$ & $-3.8 \mathrm{mas}$ & $1.0 \mathrm{mas}$ \\
\hline \multicolumn{5}{|c|}{${\text { Gaia } \text { coordinates }^{(b)}}$} \\
\hline Epoch & RA & $\sigma_{\mathrm{RA}}$ & Dec & $\sigma_{\text {Dec }}$ \\
\hline 2016.61 & $274.5245619^{\circ}$ & $0.2 \mathrm{mas}$ & $-12.2425958^{\circ}$ & $0.2 \mathrm{mas}$ \\
\hline
\end{tabular}

Notes. ${ }^{(a)}$ The reported error bars include the uncertainty reported for the position of the binary system with respect to the radio emission derived from our model, and the intrinsic astrometric error of the radio maps. ${ }^{(b)}$ The reported error bars include the Gaia uncertainty of the 2015.5 position and the uncertainties in the proper motions.

\subsection{Astrometric solution}

Our VLBI radio observations provide us with the absolute astrometric position of the bowshock and our model allowed us to locate the projected position of the binary referenced to the radio emission. In Table 2, the positions of the emission peaks of the radio maps are reported, together with the relative position of the SB from its respective radio maps (as computed from our model). We confirmed our 2016 astrometric solution by comparing the position of the system reported in the Gaia Data Release 2 archive (Gaia Collaboration 2016a,b). The Gaia 2015.5 epoch reported a RA $=274.5245621$ and $\mathrm{Dec}=-12.2425954$ (with uncertainties around 0.1 mas) for the position of HD 167971. Correcting by the barycentric proper motions $\left(\mathrm{PM}_{\mathrm{RA}}^{\mathrm{sys}}=-0.54 \pm 0.19 \mathrm{mas} \mathrm{yr}^{-1}\right.$;

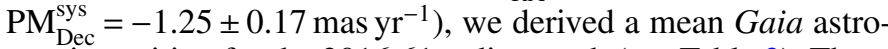
metric position for the 2016.61 radio-epoch (see Table 2). These new coordinates set the Gaia astrometric solution in between the line that connects the SB and the T, confirming the astrometry derived from our VLTI binary fit and VLBA radio emission (see Fig. 4).

\section{Conclusions}

In this paper we have shown that the nonthermal radio emission observed in the $\mathrm{C}$ and $\mathrm{X}$ bands is in agreement with the wind-wind collision region between the SB and the more distant $\mathrm{T}$ in HD 167971. The morphology of the emission changes in accordance with the predicted orbital motion of the components as determined from NIR interferometric observations, with the semi-major axis perpendicular to the projected line that connects the SB and the T across the orbital path. The total intensity also changes accordingly between the two observing epochs in a way inversely proportional to the separation of the stellar components in the long-period system, with a steep spectral index of $\alpha \sim-1.1$. Since the WWCR is outside the radio photosphere of both the SB and T for all the orbital path, freefree absorption and the Razin effect are excluded as attenuating mechanisms of the synchrotron emission at the observed frequencies. In contrast, inverse Compton cooling appears to play an important role in the steepness of $\alpha$. Our model allowed us to locate the orbital components relative to the bowshock position, finding an absolute astrometric solution for the 
system, which in turn was confirmed with an independent Gaia measurement.

This work presents a first step towards understanding the physics of the observed synchrotron radio emission of HD 167971. It shows the importance of VLBI observations to characterize the wind-wind interaction region in the system. Future multi-wavelength VLBI radio observations are necessary to fully characterize the emission across the different orbital phases. A monitoring campaign is needed to constrain the stellar parameters of the components of HD 167971 and to predict, through radiative transfer modelling, its evolution. Complementary observations with the high-resolution $(R \sim 4000)$ mode of GRAVITY/VLTI are also envisioned to characterize possible infrared shock tracers (like $\mathrm{Br} \gamma$ ) correlated with the observed WWCR at radio frequencies (see e.g., Sanchez-Bermudez et al. 2017; Gravity Collaboration 2018). HD 167971 is a unique target that can bring us new insights into the synchrotron emission in multiple O-star systems. Understanding the properties of the stars could help us to better characterize more distant targets in high-mass star forming regions (e.g., the stellar radio emitters in W51; Ginsburg et al. 2016), which in turn would provide important constraints on the radiation and chemical feedback of massive stars.

Acknowledgements. We thank the anonymous referee for the very useful comments. J.S.B acknowledges support from the ESO Fellowship program. This work made use of data from the European Space Agency (ESA) mission Gaia (https://www.cosmos.esa.int/gaia), processed by the Gaia Data Processing and Analysis Consortium (DPAC, https://www.cosmos.esa.int/ web/gaia/dpac/consortium). Funding for the DPAC has been provided by national institutions, in particular the institutions participating in the Gaia Multilateral Agreement. The research leading to these results has received funding from the European Research Council under the European Union's Seventh Framework Programme (FP7/2007-2013)/ERC grant agreement no. [614922]. A.A., M.P.-T. acknowledge support from the Spanish MINECO through grant AYA2015-63939-C2-1P, cofunded with FEDER funds. J.C.G., and J.M.M. were partially supported by the Spanish MINECO project AYA2015-63939-C2-2P. R.G.M. and J.S.B. acknowledge support from UNAM-PAPIIT Programme IN104319.

\section{References}

Benaglia, P., \& Koribalski, B. 2007, in Massive Stars in Interactive Binaries, eds. N. St.-Louis, \& A. F. J. Moffat, ASP Conf. Ser., 367, 179

Bieging, J. H., Abbott, D. C., \& Churchwell, E. B. 1989, ApJ, 340, 518

Blomme, R., \& Volpi, D. 2014, A\&A, 561, A18

Blomme, R., van Loo, S., De Becker, M., et al. 2005, A\&A, 436, 1033

Blomme, R., De Becker, M., Runacres, M. C., van Loo, S., \& Setia Gunawan, D. Y. A. 2007, A\&A, 464, 701

Canto, J., Raga, A. C., \& Wilkin, F. P. 1996, ApJ, 469, 729

De Becker, M., Rauw, G., Blomme, R., et al. 2004, A\&A, 420, 1061

De Becker, M., Rauw, G., Blomme, R., et al. 2005, A\&A, 437, 1029

De Becker, M., Sana, H., Absil, O., Le Bouquin, J.-B., \& Blomme, R. 2012, MNRAS, 423, 2711

Dougherty, S. M., \& Williams, P. M. 2000, MNRAS, 319, 1005

Dougherty, S. M., Williams, P. M., van der Hucht, K. A., Bode, M. F., \& Davis, R. J. 1996, MNRAS, 280, 963

Dougherty, S. M., Pittard, J. M., Kasian, L., et al. 2003, A\&A, 409, 217

Eichler, D., \& Usov, V. 1993, ApJ, 402, 271

Foreman-Mackey, D., Hogg, D. W., Lang, D., \& Goodman, J. 2013, PASP, 125, 306

Gaia Collaboration (Brown, A. G. A., et al.) 2016a, A\&A, 595, A12

Gaia Collaboration (Prusti, T., et al.) 2016b, A\&A, 595, A1

Gayley, K. G. 2009, ApJ, 703, 89

Ginsburg, A., Goss, W. M., Goddi, C., et al. 2016, A\&A, 595, A27

Gravity Collaboration, Sanchez-Bermudez, J., Weigelt, G., et al. 2018, A\&A, 618, A125

Ibanoglu, C., Çakırlı, Ö., \& Sipahi, E. 2013, MNRAS, 436, 750

Le Bouquin, J.-B., Sana, H., Gosset, E., et al. 2017, A\&A, 601, A34

Leitherer, C., Forbes, D., Gilmore, A. C., et al. 1987, A\&A, 185, 121

Montes, G., Pérez-Torres, M. A., Alberdi, A., \& González, R. F. 2009, ApJ, 705, 899

Montes, G., Alberdi, A., Pérez-Torres, M. A., \& González, R. F. 2015, Rev. Mex. Astron. Astrofis., 51, 209

Pittard, J. M., \& Dawson, B. 2018, MNRAS, 477, 5640

Pittard, J. M., \& Dougherty, S. M. 2006, MNRAS, 372, 801

Pittard, J. M., Dougherty, S. M., Coker, R. F., O'Connor, E., \& Bolingbroke, N. J. 2006, A\&A, 446, 1001

Rauw, G. 2004, in Cosmic Gamma-Ray Sources, eds. K. S. Cheng, \& G. E. Romero, Astrophys. Space Sci. Lib., 304, 105

Sanchez-Bermudez, J., Alberdi, A., Barbá, R., et al. 2017, ApJ, 845, 57

van Loo, S., Blomme, R., Dougherty, S. M., \& Runacres, M. C. 2008, A\&A, 483,585

White, R. L., \& Becker, R. H. 1995, ApJ, 451, 352

Williams, P. M., van der Hucht, K. A., The, P. S., \& Bouchet, P. 1990, MNRAS, 247,18 


\section{Appendix A: HD 167971 spectral index map}

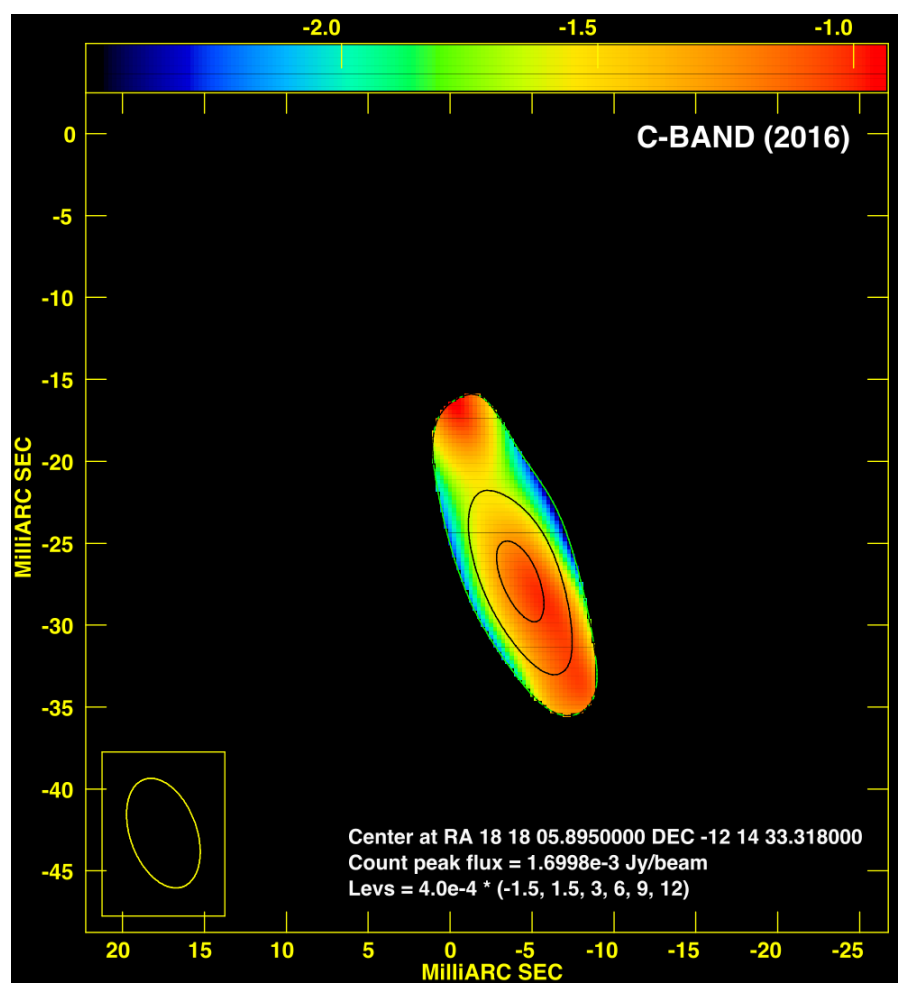

Fig. A.1. Spectral index of HD 167971 between $X$-band and $C$-band of the 2016 epoch is represented in colour. In contours (representing $30,50,70$, and $90 \%$ of the peak) the image displays the structure of HD 167971 observed at the $X$-band. We note how the index across the whole structure is quite consistent around $\alpha=-1.1$, confirming the nonthermal emission of the observed radio emission.

\section{Appendix B: Bowshock profiles}

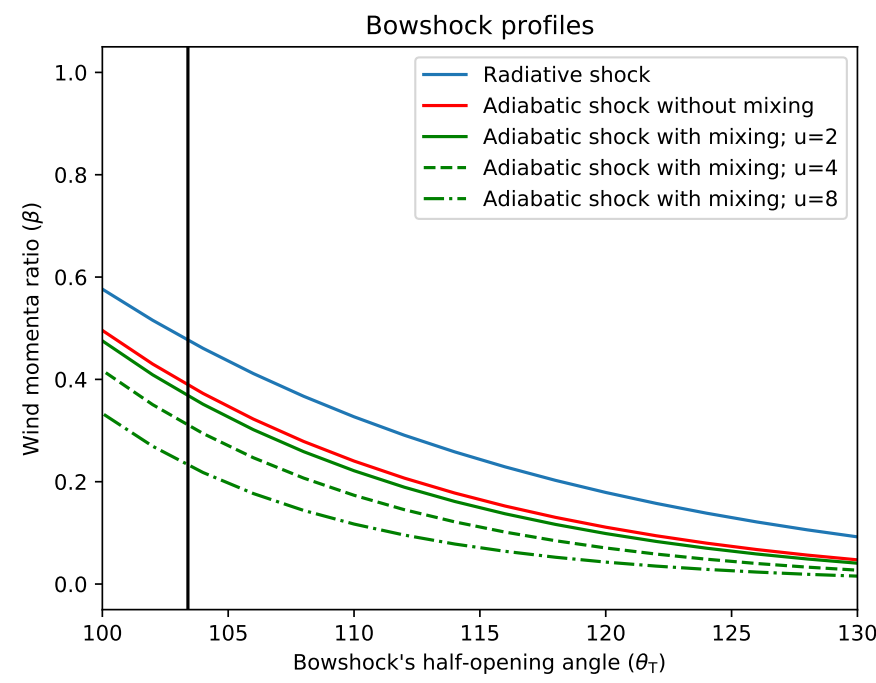

Fig. B.1. Value of $\beta$ as a function of the half-opening angle $\left(\theta_{\mathrm{T}}\right)$ of the bowshock profile for different types of wind-wind collision shocks according to Canto et al. (1996) (radiative) and Gayley (2009) (adiabatic). The labels on the frame describe each of the different shock types plotted. In the case of the adiabatic shock with mixing, three different cases are shown for different values of terminal velocity ratio, $u$. The vertical black solid line shows the corresponding $\beta$ values $\theta_{\mathrm{T}}=103.4^{\circ}$, which is the best-fit value for the shock profile obtained from our 2006 $C$-band image. 